\title{
LIMITACIONES \\ CONSTITUCIONALES QUE AFECTAN A LA ELECCIÓN DEL CONGRESO DE LOS DIPUTADOS. POSIBLE MODIFICACIÓN DEL ARTÍCULO 68 DE LA CONSTITUCIÓN Y REPARTO A DOS NIVELES
}

VICTORIANO RAMÍREZ GONZÁLEZ 
0. INTRODUCCIÓN. 1. LIMITACIONES CONSTITUCIONALES ACTUALES. 2. LEY ELECTORAL Y PRINCIPALES CONTRADICCIONES EN LOS RESULTADOS GLOBALES EN LAS ELECCIONES DEL CONGRESO DE LOS DIPUTADOS. 3. VOTOS QUE NO HAN DADO LUGAR A REPRESENTACIÓN EN LA ELECCIÓN DE 2004. 4. POSIBILIDADES DE REFORMA DE LA LEY ELECTORAL DENTRO DEL MARCO CONSTITUCIONAL ACTUAL. 5. REPARTO A DOS NIVELES. LA ELECCIÓN DEL BUNDESTAG EN ALEMANIA. 6. ¿QUÉ RESULTADOS SE HABRÍAN PODIDO OBTENER EN LA ELECCIÓN DEL CONGRESO EN 2004 USANDO UN REPARTO A SEGUNDO NIVEL? 7. MODIFICACIÓN DEL ARTÍCULO 68 DE LA CONSTITUCIÓN. REFERENCIAS 


\title{
LIMITACIONES CONSTITUCIONALES QUE AFECTAN A LA ELECCIÓN DEL CONGRESO DE LOS DIPUTADOS. POSIBLE MODIFICACIÓN DEL ARTÍCULO 68 DE LA CONSTITUCIÓN Y REPARTO A DOS NIVELES
}

POR

\author{
VICTORIANO RAMÍREZ GONZÁLEZ \\ Catedrático de Matemática Aplicada \\ Universidad de Granada
}

\section{INTRODUCCIÓN}

Las circunscripciones para la elección del Congreso de los Diputados son las 50 provincias y cada una de las poblaciones de Ceuta y Melilla. Los escaños de cada circunscripción se asignan a los partidos que concurren en ella en proporción a los votos de sus listas. En circunscripciones medianas y pequeñas muy pocos partidos reciben escaños con el sistema electoral actual.

Normalmente, al efectuar el reparto en una circunscripción pequeña sólo un partido (rara vez dos) recibe más escaños de su cuota, es el partido beneficiado en el reparto. Los restantes partidos reciben menos escaños de su cuota. O el partido que más votos obtiene, o bien el se- 
gundo más votado, es el que suele recibir más escaños de su cuota. La mayoría de los partidos no obtiene ningún escaño (sea cual sea el tamaño de la circunscripción) y, por tanto, pierde los votos recibidos en esa circunscripción, ya que con el sistema electoral actual los votos recibidos por un partido en una circunscripción sólo se tienen en cuenta al hacer el reparto de los escaños de esa circunscripción.

Como consecuencia, aunque los repartos en las circunscripciones se hagan con un método proporcional, la desigual pérdida de votos de unos partidos frente a otros produce una desproporción importante entre votos y escaños totales recibidos por los partidos. Pues hay partidos que son perjudicados en todas (o en casi todas) las circunscripciones, mientras que otros pueden ser beneficiados, aun teniendo menos votos que aquellos, porque en las circunscripciones que concurren consiguen estar entre los más votados. Ello hace que, en la elección del Congreso de los Diputados, existan comparaciones paradójicas y por tanto agravios importantes entre los resultados de unos partidos y otros. Actualmente, el valor del voto de los ciudadanos depende en gran medida de la circunscripción donde se emita o del partido al que se le otorgue. Lejos está el principio democrático de: "una persona un voto».

Otra consecuencia es que el sistema electoral permite que un partido gane las elecciones en número de votos y otro sea el que más escaños reciba.

Una posible solución se obtendría con una ligera disminución de las limitaciones establecidas en el artículo 68 de la Constitución, que permitiría diseñar un sistema electoral que contemplase un reparto a varios niveles, o un reparto biproporcional, (métodos bastante más lógicos y más justos que el sistema actual). Con cualquiera de ellos se reducirían los agravios que produce el sistema electoral actual. Más adelante veremos cómo puede aplicarse el reparto a varios niveles, por ser el más sencillo de ambos.

Previamente, en los apartados 1 y 2 de este trabajo se recogen las principales limitaciones constitucionales que afectan al sistema electoral del Congreso de los Diputados y se resaltan las contradicciones más importantes que, en cada elección de las celebradas entre 1977 y 2004 , se han producido, al comparar votos totales y escaños totales de algunos partidos políticos [9]. Se observa que, sistemáticamente, algún partido de ámbito estatal es perjudicado con respecto a otro de ámbito autonómico.

En cada circunscripción, muchos votos son emitidos a favor de partidos que no consiguen representación en ella, hay quien los denomina 
"votos no útiles"; un recuento de los mismos para la elección de 2004 aparece en el apartado 3. Estos «votos no útiles» suelen ser más de dos millones en cada elección. Es decir, entorno al $10 \%$ de los votos válidos a favor de candidaturas suelen ser "no útiles" con el sistema electoral actual. La ley electoral de 1985 podría cambiarse [3,7], como se indica en el apartado 4 (sin modificar la Constitución), de forma que tales votos disminuyan, pero aún así seguirá habiendo muchos votos no útiles, agravios importantes entre partidos de ámbito estatal y de ámbito autonómico (con un número similar de votos) y seguirá siendo muy difícil la aparición de nuevos partidos de ámbito estatal.

La técnica basada en reparto de los escaños a dos niveles permite conseguir una gran equidad en el valor del voto del elector, independientemente del lugar donde se emita y del partido al que se otorgue (siempre que no sea un partido muy pequeño) [4]. Esta técnica se comenta en el apartado 5, y se muestra una aplicación de la misma: la elección del Bundestag en Alemania en 2002.

A continuación, en el apartado 6, se muestra una simulación de reparto a dos niveles para la elección del Congreso de los Diputados con los datos de 2004. Finalmente se indica una posible modificación del artículo 68 de la Constitución que permite realizar repartos a dos niveles y conseguir la mayoría de las bondades del sistema electoral alemán, manteniendo el espíritu de la Constitución de 1978.

En el anexo están los resultados, a nivel de votos y escaños globales obtenidos en las nueve elecciones celebradas desde 1977 a 2004, [9].

\section{LIMITACIONES CONSTITUCIONALES ACTUALES}

Las principales limitaciones que establece la Constitución de 1978, en su artículo 68, con respecto a la elección del Congreso de los Diputados, son:

i. el Congreso tendrá entre 300 y 400 diputados,

ii. las circunscripciones electorales serán las provincias y las poblaciones de Ceuta y Melilla. Cada provincia recibirá una asignación inicial y el resto de los escaños se distribuirán en proporción a los habitantes de las provincias (salvo Ceuta y Melilla que estarán representadas por un diputado cada una),

iii. la asignación de los escaños a los partidos se hará, en cada provincia, atendiendo a criterios de representación proporcional. 


\section{Consecuencias}

La Constitución obliga a que la circunscripción electoral sea la provincia, (además Ceuta y Melilla son circunscripciones uninominales), y a que cada provincia reciba un número inicial de escaños.

El hecho de decir que se asignará un número fijo de escaños a cada provincia y el resto se distribuirán en proporción a la población debe entenderse que ese mínimo será al menos de un diputado. La Ley Electoral ha establecido que el mínimo sea el mismo para todas las provincias; de tal forma que en ese reparto reciben la misma representación los habitantes de una provincia con menos de 200,000 habitantes que los habitantes de Madrid o Barcelona que son más de cinco millones en cada caso.

El segundo reparto a las circunscripciones corrige en gran medida esa desproporción, ya que se hace de acuerdo con el número de habitantes de cada provincia. Aún así, finalmente por cada 160,000 habitantes de una provincia grande existirá un representante, mientras que en una de las provincias pequeñas será por cada 40,000 habitantes (incluso menos). Una desigualdad en el valor del voto de los ciudadanos que puede disminuirse [5].

Sin embargo, no es el tamaño de las circunscripciones la causa principal de las paradojas que surgen cuando comparamos los votos totales y los escaños totales recibidos por los diferentes partidos, sino el hecho de realizar los repartos a los partidos en cada circunscripción de forma independiente; pues eso significa que los partidos de ámbito nacional tercero y siguientes no obtienen escaño alguno en la mayoría de las provincias, no siéndole útiles esos votos y originando agravios importantísimos al comparar los resultados de estos partidos con los correspondientes a partidos de ámbito autonómico, como vemos a continuación.

\section{LEY ELECTORAL Y PRINCIPALES CONTRADICCIONES EN LOS RESULTADOS GLOBALES EN LAS ELECCIONES DEL CONGRESO DE LOS DIPUTADOS}

La Ley Electoral de 1985 establece que el mínimo que debe asignarse a cada provincia es de dos escaños y que la fórmula para la asignación de los escaños en cada provincia debe ser el método d'Hondt. La fórmula d'Hondt se había aplicado también en las tres convocatorias anteriores a 1985 y la asignación de los escaños a las 
provincias en esas elecciones había sido equivalente a la descrita en la Ley Electoral de 1985.

La aplicación de dicha ley, en las nueve elecciones celebradas entre 1977 y 2004, ha originado muchos agravios, a nivel de votos totales y escaños totales, siendo los más importantes los que aparecen en la Tabla 1.

TABLA 1. Principales agravios en la elección del Congreso de los Diputados

\begin{tabular}{|l|l|l|c|c|c|}
\hline Año & Partido & \multicolumn{1}{|c|}{ Ámbito } & Puesto & Votos totales & Escaños totales \\
\hline 1977 & PSP & Nacional & $\mathbf{5 .}^{\circ}$ & 816,510 & $\mathbf{6}$ \\
& PDC & Comunidad Aut. & $6 .^{\circ}$ & 514,647 & 11 \\
\hline 1979 & CD & Nacional & $\mathbf{5 .}^{\circ}$ & $1,064,333$ & $\mathbf{9}$ \\
& CiU & Comunidad Aut. & $5 .^{\circ}$ & 483,254 & 9 \\
\hline 1982 & UCD & Nacional & $\mathbf{3 .}^{\circ}$ & $1,354,858$ & $\mathbf{1 1}$ \\
& PCE & Nacional & $\mathbf{4 .}^{\circ}$ & 844,976 & $\mathbf{4}$ \\
& CiU & Comunidad Aut. & $5^{\circ}$ & 772,726 & 12 \\
\hline 1986 & IU & Nacional & $5^{\circ}$ & 768,158 & $\mathbf{6}$ \\
& PNV & Comunidad Aut. & $6^{\circ}$ & 309,610 & 6 \\
\hline 1989 & IU & Nacional & $3^{\circ}$ & $1,851,080$ & $\mathbf{1 7}$ \\
& CiU & Comunidad Aut. & $5^{\circ}$ & $1,030,476$ & 18 \\
\hline 1993 & CDS & Nacional & $5^{\circ}$ & 414,000 & $\mathbf{0}$ \\
& PNV & Comunidad Aut. & $6^{\circ}$ & 300,308 & 5 \\
\hline 1996 & IU & Nacional & $\mathbf{3 .}^{\circ}$ & $2,639,774$ & $\mathbf{2 1}$ \\
& CiU & Comunidad Aut. & $4 .^{\circ}$ & $1,151,633$ & 16 \\
\hline 2000 & IU & Nacional & $\mathbf{3 .}^{\circ}$ & $1,263,043$ & $\mathbf{8}$ \\
& CiU & Comunidad Aut. & $4 .^{\circ}$ & 970,421 & 15 \\
\hline 2004 & IU & Nacional & $\mathbf{3 .}^{\circ}$ & $1,284,081$ & $\mathbf{5}$ \\
& ERC & Comunidad Aut. & $5^{\circ}$ & 652,196 & 8 \\
\hline
\end{tabular}

\section{Consecuencia}

En todas las elecciones, un partido de ámbito estatal (normalmente el tercero y cualquiera de los siguientes) ha sido perjudicado con respecto a los partidos de ámbito autonómico similares en número de votos.

Así, de forma sistemática IU (o PCE en las primeras convocatorias) ha sido uno de ellos y, en cada elección, ha necesitado entre el doble y el cuádruplo de votos por escaño que la media de los restantes partidos con representación parlamentaria. Algo muy lejano de los principios de igualdad establecidos en la Constitución (artículos 1.1, 9.2,14) y de democracia avanzada (preámbulo). 
Como consecuencia de ello, resulta bastante significativo el hecho de que después de 1982 no haya surgido ningún partido de ámbito estatal que haya conseguido representación parlamentaria; además, las posibilidades de que eso ocurra, sin cambiar el sistema electoral actual, son mínimas.

El sistema electoral actual es muy injusto con todos los partidos de ámbito estatal excepto con los dos primeros. Además, si se produce un descenso en votos de uno de los dos primeros partidos de ámbito estatal también puede llevar a ese partido a un coste de los escaños muy superior al coste (votos/escaños) de los partidos de ámbito autonómico.

\section{VOTOS QUE NO HAN DADO LUGAR A REPRESENTACIÓN EN LA ELECCIÓN DE 2004}

Si contabilizamos el número de votos que, en cada circunscripción, corresponden a partidos que no consiguieron representación en ella, los resultados para la elección de 2004 son los siguientes:

TABLA 2. Votos que no condujeron a representación en 2004

\begin{tabular}{|l|l|c|}
\hline \multicolumn{1}{|c|}{ Partido } & \multicolumn{1}{|c|}{ Circunscripciones } & Votos no útiles \\
\hline PSOE & Ceuta y Melilla & 24,042 \\
\hline PP & Girona y Lleida & 75,075 \\
\hline IU & $\begin{array}{l}\text { Todas excepto: } \\
\text { Madrid, Barcelona y Valencia }\end{array}$ & 782,341 \\
\hline BNG & Lugo y Ourense & 51,466 \\
\hline CHA & Huesca y Teruel & 13,092 \\
\hline EA & Álava y Vizcaya & 37,934 \\
\hline $\begin{array}{l}\text { Partidos sin } \\
\text { representación }\end{array}$ & Todas & $1,229,153$ \\
\hline Totales & & $2,213,103$ \\
\hline
\end{tabular}

Más de dos millones doscientos mil ciudadanos pudieron comprobar la noche electoral del 14 de Marzo de 2004 que el partido al cual habían votado no había conseguido representación en su circunscripción. Fueron muchos los votantes desencantados. En otras convocatorias electorales anteriores, cuando existía algún partido más de ámbito nacional, los votos que no obtuvieron representación llegaron a pasar de 
dos millones y medio. Normalmente, suelen ser alrededor del $10 \%$ de los votos emitidos en cualquiera de las elecciones al Congreso de Diputados.

\section{POSIBILIDADES DE REFORMA DE LA LEY ELECTORAL DE 1985 DENTRO DEL MARCO CONSTITUCIONAL ACTUAL}

En el supuesto de que se desee una mayor equidad en el valor del voto de cada ciudadano, independientemente de su lugar de residencia y del partido al que vote (siempre que dicho partido no sea muy minoritario), sin modificar la Constitución, las posibilidades de reforma son $[3,7,8]$ :

i. Disminuir la asignación inicial de cada provincia a un escaño.

ii. Sustituir el método d'Hondt (que beneficia al partido más votado en cada circunscripción) por otro que no beneficie al partido más votado en cada circunscripción, por ejemplo, por Sainte Laguë (que es una fórmula imparcial) [1].

iii. Aumentar el tamaño del Congreso a 400.

Con estos cambios el efecto que se produce es el siguiente:

a. Disminuyen los agravios entre los partidos de ámbito autonómico más destacados y el tercer partido de ámbito estatal.

b. Aumenta la proporcionalidad.

c. Se dificulta la gobernabilidad, ya que el aumento de representación de partidos tercero y siguientes de ámbito estatal sería principalmente a costa de los dos primeros partidos de ámbito estatal.

d. Hay más votos útiles, pero aún seguirá habiendo muchos votos que no conduzcan a representación.

En cualquier caso, sin modificar la Constitución, no se podrán aplicar técnicas de investigación recientes para efectuar las asignaciones de los escaños, como por ejemplo: repartos biproporcionales [2] (aplicado por primera vez el 12-02-2006 en Zurich), ni tampoco se podrán aplicar repartos a varios niveles (como hace el sistema electoral alemán). Ambos métodos dan lugar a resultados mucho más justos que el sistema electoral actual. 


\section{REPARTO A DOS NIVELES. LA ELECCIÓN DEL BUNDESTAG EN ALEMANIA}

Algunos sistemas electorales realizan repartos a varios niveles. Por ejemplo, uno de ellos es el sistema electoral para la elección del Bundestag en Alemania.

El sistema electoral para la elección del Bundestag alemán es uno de los mejores del mundo en el momento actual. Efectivamente los porcentajes de participación, el índice de proporcionalidad, la equidad en el valor de los votos, etc., difícilmente son superados en ningún otro país. Tal vez es el tipo de barrera establecido la característica más discutible, y casi seguro mejorable.

El tamaño del Bundestag es de 598 escaños, de los cuales la mitad ellos, 299, son asignados al partido más votado en cada uno de los 299 distritos uninominales existentes. Este es el primer nivel de reparto. A continuación se realiza un reparto de los 598 escaños (los 299 ya asignados más otros 299 añadidos) entre los partidos que han obtenido al menos el $5 \%$ de los votos o bien han conseguido al menos tres escaños en los distritos uninominales. Es el reparto a segundo nivel. Al realizar el segundo reparto cada partido debe recibir al menos los escaños que ya había ganado en los distritos uninominales.

Casi nunca es necesario considerar esos mínimos a nivel nacional porque todos los partidos aumentan, ya que son muchos los escaños añadidos para el reparto a segundo nivel. No obstante, en alguna ocasión, como ha ocurrido en las elecciones de 2002, para que ningún partido recibiera menos de los escaños que le correspondían en el segundo nivel en cada Estado Federal, ha sido necesario ampliar el tamaño de la Cámara.

Concretamente el tamaño del Bundestag en 2002 ha sido de 603 diputados, distribuidos de la siguiente forma [6]:

TABLA 3. Elección del Bundestag en 2002

\begin{tabular}{|l|r|c|c|c|c|}
\hline Partido & Votos & \%votos & Esc.-Mayoría & Esc.-Propor. & Total \\
\hline SPD & $18,488,668$ & 38.50 & 171 & 247 & $\mathbf{2 4 7 + 4}$ \\
\hline CDU & $14,167,561$ & 29.52 & 82 & 189 & $\mathbf{1 8 9 + 1}$ \\
\hline CSU & $4,315,080$ & 8.99 & 43 & 58 & $\mathbf{5 8}$ \\
\hline GRÜNE & $4,110,355$ & 8.56 & 1 & 55 & $\mathbf{5 5}$ \\
\hline FDP & $3,538,815$ & 7.37 & 0 & 47 & $\mathbf{4 7}$ \\
\hline PDS & $1,916,702$ & 3.99 & 2 & - & $\mathbf{2}$ \\
\hline Total & $46,537,181$ & 96.93 & 299 & 598 & $\mathbf{5 9 8 + 5}$ \\
\hline
\end{tabular}


EI SPD obtuvo en la elección por mayoría un diputado más de los que le correspondía en proporción a sus votos totales en cuatro estados (Bayern, Baden-Württemberg, Sachsen y Nordrhein-Westfalen) y el CDU en uno (Sachsen-Anhalt). Por eso el resultado final de la Cámara contiene cinco escaños más de los previstos.

Evidentemente la opción de no fijar el tamaño de la Cámara, permitiendo que pueda aumentar debido a que a algún partido le corresponda un número total de escaños inferior al obtenido en el reparto a primer nivel, es una opción tan aceptable como la de realizar el reparto a segundo con mínimos aplicables a los Estados Federales.

Como se observa, en la Tabla 3, el único agravio importante en los resultados globales de los partidos se produce entre el PDS que no supera la barrera del $5 \%$ y cualquiera de los partidos que ligeramente superan esa barrera: FDP, GRÜNE y CSU. De ahí el interés en usar barreras continuas, en lugar de las barreras clásicas; pues con una barrera continua de 500,000 votos restados a los votos de cada partido (equivale a restar algo más del $1 \%$ de los votos totales), el reparto que se obtiene es:

SPD-248, CDU-189, CSU-52, GRÜNE-49, FDP-41, PDS-19

que no dificulta la gobernabilidad a ninguno de los dos grandes partidos y es más justo para los cuatro partidos medianos.

Con el sistema electoral del Bundestag todos los votos de un mismo partido suelen tener un valor idéntico. Eso induce a la participación. Así la participación en las diferentes elecciones celebradas en Alemania desde el 14 de Agosto de1949 ha sido la siguiente:

TABLA 4. Porcentajes de participación en las elecciones del Bundestag

\begin{tabular}{|cccc|}
\hline Elección & Participación & Elección & Participación \\
\hline 1949 & 91.3 & 1953 & 93.6 \\
\hline 1957 & 93.0 & 1961 & 94.3 \\
\hline 1965 & 96.4 & 1969 & 94.5 \\
\hline 1972 & 99.1 & 1976 & 99.1 \\
\hline 1980 & 98.0 & 1983 & 99.5 \\
\hline 1987 & 98.6 & 1990 & 91.9 \\
\hline 1994 & 96.4 & 1998 & 94.1 \\
\hline 2002 & 93.0 & 2006 & $¿ ?$ \\
\hline
\end{tabular}


En todos los casos superó el $91 \%$, con una media del 95.5\%. Sin embargo, la participación en las elecciones al Congreso en España ha sido la siguiente:

TABLA 5. Porcentajes de participación en las elecciones del Congreso

\begin{tabular}{|cccc|}
\hline Elección & Participación & Elección & Participación \\
\hline 1977 & 78.83 & 1979 & 68.04 \\
\hline 1982 & 79.97 & 1986 & 70.49 \\
\hline 1989 & 69.74 & 1993 & 76.44 \\
\hline 1996 & 77.38 & 2000 & 68.71 \\
\hline 2004 & 75.66 & & \\
\hline
\end{tabular}

Siempre inferior al $80 \%$ y la media es: $73.92 \%$. Es decir, en media, la participación en las elecciones al Congreso de los Diputados en España ha sido 21 puntos por debajo de la participación en Alemania para la elección del Bundestag.

\section{6. ¿QUÉ RESULTADOS SE HABRÍAN PODIDO OBTENER EN LA ELECCIÓN DEL CONGRESO EN 2004 USANDO UN REPARTO A SEGUNDO NIVEL?}

Pera realizar un reparto a dos niveles no es necesario imitar de forma exacta el sistema electoral alemán. Hay multitud de formas, que permiten adaptarse a las peculiaridades de cada país, para conseguir alcanzar muchas de las bondades de ese sistema electoral.

Así por ejemplo, en España una vez que se ha realizado la asignación de escaños en las circunscripciones actuales para la elección del Congreso, es posible hacer un nuevo reparto de escaños en el que se añaden nuevos escaños a los 350 ya asignados a los partidos y se hace un reparto de la totalidad de los escaños (la suma de los ya asignados y los añadidos) en proporción a los votos totales de los partidos. Este es el reparto a segundo nivel.

Con un reparto a segundo nivel se puede conseguir que:

i. El valor del voto de un partido sea el mismo, independientemente de que lo reciba en una circunscripción u otra.

ii. El número de votos llamados "no útiles» descienda bruscamente. 
iii. La proporcionalidad sea muy elevada.

iv. Aumente la participación.

Es decir, sin necesidad de recurrir a elecciones uninominales en distritos, como hace Alemania, se pueden conseguir resultados muy similares.

Lógicamente, si no se ponen restricciones para el reparto a segundo nivel, es posible que a un partido le correspondan menos escaños de los que ya se le habían asignado en las circunscripciones al realizar el primer reparto. Para que eso no ocurra hay que imponer unos mínimos de representación para el reparto a segundo nivel. Por tanto, el mínimo número de escaños que se asignará a cada partido, en el reparto a nivel de votos totales, debe ser el número total de escaños obtenidos por ese partido en las circunscripciones en el primer reparto.

A modo de ejemplo, con objeto de poder simular algunos resultados, vamos a suponer que el sistema electoral del Congreso contemplara dos niveles de asignación de escaños a los partidos. Supongamos también que el reparto a primer nivel es idéntico al actual y que el reparto a segundo nivel se hace para un tamaño total de 400 escaños (se añaden sólo 50 escaños a los 350 iniciales), con mínimos iguales a los escaños obtenidos en el primer reparto.

La barrera a usar va a ser de tipo continuo, concretamente supongamos que se restan a los votos totales de cada partido 300,000; que equivale a algo más del $1 \%$ de los votos emitidos a candidaturas. Barrera algo baja. Los partidos que tengan menos de 300,000 votos no participan en el reparto a segundo nivel. Con esta barrera, cualquier partido con el $1.5 \%$ de los votos totales obtiene representación en el Congreso, (en Alemania los partidos deben alcanzar el 5\% para participar en el segundo reparto).

Entonces el reparto obtenido sería el que aparece en la Tabla 6.

En la tabla 7 podemos observar que el sistema electoral actual origina grandes diferencias, en el coste de votos por escaños, entre unos partidos y otros $y$, además, el partido vencedor de las elecciones no es el más beneficiado.

Sin embargo, la misma tabla muestra que la relación votos/escaños es bastante más similar con el reparto a dos niveles realizado. En este caso, el partido que consigue los escaños a menor coste es el vencedor de las elecciones. 
TABLA 6. Asignación a dos niveles con los datos de 2004 del Congreso

\begin{tabular}{|l|r|r|r|r|r|c|}
\hline Partido & $\begin{array}{c}\text { Votos } \\
\text { Totales }\end{array}$ & $\begin{array}{c}\% \\
\text { Votos }\end{array}$ & $\begin{array}{c}\text { Reparto } \\
\text { actual }\end{array}$ & $\begin{array}{c}\text { Votos } \\
\text { Reducidos }\end{array}$ & Mínimo & $\begin{array}{c}\text { Asignación } \\
\text { a dos niveles }\end{array}$ \\
\hline PSOE & $11,026,163$ & 44.38 & 164 & $10,726,163$ & 164 & $\mathbf{1 8 6}$ \\
\hline PP & $9,763,144$ & 39.30 & 148 & $9,463,144$ & 148 & $\mathbf{1 6 4}$ \\
\hline IU & $1,284,081$ & 5.17 & 5 & 984,081 & 5 & $\mathbf{1 7}$ \\
\hline CiU & 835,471 & 3.36 & 10 & 535,471 & 10 & $\mathbf{1 0}$ \\
\hline ERC & 652,196 & 2.62 & 8 & 352,196 & 8 & $\mathbf{8}$ \\
\hline PNV & 420,980 & 1.69 & 7 & 120,980 & 7 & $\mathbf{7}$ \\
\hline CC & 235,221 & 0.95 & 3 & 0 & 3 & $\mathbf{3}$ \\
\hline BNG & 208,688 & 0.84 & 2 & 0 & 2 & $\mathbf{2}$ \\
\hline PA & 181,868 & 0.73 & 0 & 0 & 0 & $\mathbf{0}$ \\
\hline CHA & 94,252 & 0.38 & 1 & 0 & 1 & $\mathbf{1}$ \\
\hline EA & 80,905 & 0.33 & 1 & 0 & 1 & $\mathbf{1}$ \\
\hline Na-Bai & 61,045 & 0.25 & 1 & 0 & 1 & $\mathbf{1}$ \\
\hline Totales & $24,844,014$ & 100 & 350 & $22,182,054$ & 350 & 400 \\
\hline
\end{tabular}

TABLA 7. Coste de los escaños con sistema actual y con un reparto a segundo nivel en 2004

\begin{tabular}{|c|c|c|c|c|c|}
\hline Partido & $\begin{array}{l}\text { Votos } \\
\text { Totales }\end{array}$ & $\begin{array}{c}\text { Reparto } \\
\text { actual }\end{array}$ & $\begin{array}{l}\text { Votos/Escaños } \\
\text { Con rep. actual }\end{array}$ & $\begin{array}{c}\text { Reparto a } \\
\text { dos niveles }\end{array}$ & $\begin{array}{l}\text { Votos/Escaños } \\
\text { Rep. dos niveles }\end{array}$ \\
\hline PSOE & $11,026,163$ & 164 & 67,233 & 186 & 59,280 \\
\hline $\mathrm{PP}$ & $9,763,144$ & 148 & 65,967 & 164 & 59,531 \\
\hline IU & $1,284,081$ & 5 & 256,816 & 17 & 75,534 \\
\hline $\mathrm{CiU}$ & 835,471 & 10 & 83,547 & 10 & 83,547 \\
\hline ERC & 652,196 & 8 & 81,524 & 8 & 81,524 \\
\hline PNV & 420,980 & 7 & 60,140 & 7 & 60,140 \\
\hline $\mathrm{CC}$ & 235,221 & 3 & 78,407 & 3 & 78,407 \\
\hline $\mathrm{BNG}$ & 208,688 & 2 & 104,344 & 2 & 104,344 \\
\hline PA & 181,868 & 0 & - & 0 & - \\
\hline $\mathrm{CHA}$ & 94,252 & 1 & 94,252 & 1 & 94,252 \\
\hline $\mathrm{EA}$ & 80,905 & 1 & 80,905 & 1 & 80,905 \\
\hline Na-Bai & 61,045 & 1 & 61,045 & 1 & 61,045 \\
\hline Totals & $24,844,014$ & 350 & 70,983 & 400 & 62,110 \\
\hline
\end{tabular}


Para completar la simulación de un posible reparto a dos niveles, indiquemos que los nuevos escaños recibidos por cada partido como consecuencia del segundo reparto, pueden asignarse a los candidatos de las listas provinciales de ese partido. De nuevo es una asignación con requisitos mínimos, a las listas de ese partido.

Así, para los 22 escaños adicionales correspondientes al primer partido, el PSOE, tendríamos lo siguiente (reparto realizado con el método de Sainte Laguë, para no dar ventaja a los candidatos de unas provincias respecto de otras):

TABLA 8. Asignación de los escaños del PSOE correspondientes al reparto a segundo nivel

\begin{tabular}{|l|c|c|c|c|c|c|}
\hline Provincia & Votos-PSOE & Mínimo & Increm. & Asig. & Vot./Escaño & Vot./Esc-Actual \\
\hline Madrid & $1,544,676$ & 16 & 8 & 24 & 64,361 & 96,542 \\
\hline Barcelona & $1,268,028$ & 14 & 6 & 20 & 63,401 & 90,593 \\
\hline Sevilla & 639,293 & 8 & 2 & 10 & 63,929 & 79,911 \\
\hline Valencia & 613,833 & 7 & 3 & 10 & 61,383 & 87,690 \\
\hline Alicante & 374,631 & 5 & 1 & 6 & 62,438 & 74,926 \\
\hline Asturias & 305,240 & 4 & 1 & 5 & 61,048 & 76,310 \\
\hline Murcia & 252,246 & 3 & 1 & 4 & 63,061 & 84,082 \\
\hline
\end{tabular}

Las restantes provincias no aparecen porque no reciben escaños en esta asignación. El coste de votos por escaño es algo menor en las provincias más pequeñas. El coste más pequeño seguirá siendo el correspondiente a Teruel, donde el PSOE con 36,152 votos recibió 2 escaños. El coste medio actual de los escaños del PSOE es: 11,026,163/164= 67,233 votos por escaño, sin embargo en Madrid y Barcelona pasa de 90,000 (última columna de la Tabla 8). El coste medio para 400 escaños, con el reparto a dos niveles, sería: 11,026,163/186=59,280. Así pues, con el reparto a dos niveles presentado, el coste de los escaños del PSOE en todas las provincias grandes (penúltima columna de la Tabla 8) es sólo algo superior al coste medio.

Observamos que los escaños adicionales obtenidos por el PSOE en el reparto a segundo nivel son asignados a candidatos de las provincias más pobladas, es decir, a aquellas donde el coste del escaño era mayor (por estar infra-representadas). Con ello se da una representación más justa a las provincias y también a los candidatos de unas provincias frente a los de otras. 
De forma análoga se asignarían los 16 escaños del PP, que corresponderían a las provincias que aparecen en la Tabla 9.

TABLA 9. Asignación de los escaños del PP obtenidos en el reparto a segundo nivel

\begin{tabular}{|l|c|c|c|c|c|c|}
\hline Provincia & Votos-PSOE & Mínimo & Increm. & Asig. & Vot./Escaño & Vot./Esc-Actual \\
\hline Madrid & $1,576,636$ & 17 & 7 & 24 & 65,693 & 92,743 \\
\hline Valencia & 665,526 & 8 & 2 & 10 & 66,552 & 83,190 \\
\hline Barcelona & 485,504 & 5 & 3 & 8 & 60,688 & 97,100 \\
\hline Alicante & 434,812 & 6 & 1 & 7 & 62,116 & 72,468 \\
\hline Coruña & 329,389 & 4 & 1 & 5 & 65,878 & 82,347 \\
\hline Asturias & 307,977 & 4 & 1 & 5 & 61,595 & 76,994 \\
\hline Sevilla & 306,464 & 4 & 1 & 5 & 61,292 & 76,616 \\
\hline
\end{tabular}

El coste medio actual de los escaños del PP es 65,967 votos; siendo el coste menor el correspondiente a los dos escaños de Soria (que fueron obtenidos con 29,187 votos). Sin embargo el de las provincias más grandes pasó de 90,000 votos por escaño. Con el reparto a dos niveles el coste medio del escaño del PP es 59,531. Las provincias más grandes tienen un coste próximo al valor medio (penúltima columna de la Tabla 9).

Finalmente, dos de los 12 escaños adicionales de IU corresponden a Madrid y uno a cada una de las provincias siguientes: Alicante, Asturias, Barcelona, Cádiz, Córdoba, Granada, Islas Baleares, Málaga y Sevilla y Vizcaya. Como se observa en la Tabla 7, el coste medio de los escaños de IU descendería de 256,816 a 75,534 votos por escaño. IU es el partido más perjudicado con el sistema electoral actual y, por tanto, es el partido que consigue disminuir más el coste medio de los escaños con el reparto a segundo nivel.

De nuevo las provincias más pobladas y las medianas, en las que el coste del escaño ha sido mayor en el primer reparto, serían las más beneficiadas, lo cual contribuye favorablemente a mejorar su proporcionalidad en la representación de las circunscripciones. También se produce una mayor igualdad en el coste del escaño para los partidos.

Por tanto, las provincias más grandes y algunas medianas habrían aumentado su representación con las asignaciones correspondientes a los 50 escaños adicionales del reparto a segundo nivel. Concretamente, las provincias que han variado su representación son las que aparecen en la Tabla 10. 
TABLA 10. Provincias que aumentarían su representación

\begin{tabular}{|l|c|c|c|c|c|c|}
\hline Provincia & Población & \% Pobl. & Esc. Act. & \% E. Act. & $\begin{array}{c}\text { Esc. } \\
\text { Propuestos }\end{array}$ & $\begin{array}{c}\% \text { Esc. } \\
\text { Prop. }\end{array}$ \\
\hline Madrid & $5,718,942$ & 13.39 & 35 & 10.00 & 52 & 13.00 \\
\hline Barcelona & $5,052,666$ & 11.83 & 31 & 8.86 & 41 & 10.25 \\
\hline Valencia & $2,320,297$ & 5.43 & 16 & 4.57 & 21 & 5.25 \\
\hline Sevilla & $1,782,862$ & 4.17 & 12 & 3.43 & 16 & 4.00 \\
\hline Alicante & $1,632,349$ & 3.82 & 11 & 3.14 & 14 & 3.50 \\
\hline Málaga & $1,374,890$ & 3.22 & 10 & 2.86 & 11 & 2.75 \\
\hline Murcia & $1,269,230$ & 2.97 & 9 & 2.57 & 10 & 2.50 \\
\hline Cádiz & $1,155,724$ & 2.71 & 9 & 2.57 & 10 & 2.50 \\
\hline Vizcaya & $1,133,428$ & 2.65 & 9 & 2.57 & 10 & 2.50 \\
\hline Coruña & $1,120,814$ & 2.63 & 9 & 2.57 & 10 & 2.50 \\
\hline Asturias & $1,075,381$ & 2.52 & 8 & 2.29 & 11 & 2.75 \\
\hline I. Baleares & 947,361 & 2.22 & 8 & 2.29 & 9 & 2.25 \\
\hline Granada & 828,107 & 1.94 & 7 & 2.00 & 8 & 2.00 \\
\hline Córdoba & 775,944 & 1.82 & 7 & 2.00 & 8 & 2.00 \\
\hline
\end{tabular}

Obsérvese que a pesar de haber aumentado mucho su representación, con el reparto a segundo nivel, las circunscripciones más grandes aún reciben un porcentaje de escaños inferior a su cuota de población. No obstante, la representación de las provincias más pobladas es mucho más proporcional con el reparto a dos niveles realizado que con el sistema electoral actual.

El objeto de este apartado ha sido mostrar que es posible introducir mejoras en el sistema electoral actual. Las citadas anteriormente no son las únicas. Por ejemplo, con un sistema como el usado anteriormente puede surgir un partido nuevo de ámbito estatal y conseguir grupo parlamentario con que obtenga cerca del $3 \%$ de los votos, sin embargo con el sistema actual ese partido seguramente no conseguiría ni un solo representante.

De todas formas, la simulación anterior no debe entenderse como una propuesta sino como la demostración de que pueden introducirse mejoras importantes en el sistema electoral del Congreso siempre que se supriman algunas de las limitaciones establecidas en el artículo 68 de la Constitución.

Si se fuese a diseñar un sistema parecido al simulado anteriormente, basado en un reparto a dos niveles, habría que tener en cuenta que 
cada partido político usará la estrategia que más le favorezca. En tal sentido, para minimizar el efecto de la barrera continua, los partidos pequeños formarían coaliciones (como hacen en las elecciones europeas). Pues, por ejemplo, si se hubiese formado la coalición integrada por $\mathrm{CC}+\mathrm{BNG}+\mathrm{PA}+\mathrm{CHA}+\mathrm{EA}$ habría sumado más de 800,000 votos (supuesto que los votos de la coalición fueran la suma de los votos de cada partido) con lo que esa coalición participaría en el reparto a segundo nivel y ganaría escaños adicionales a los 7 obtenidos en las circunscripciones provinciales. Otra estrategia sería la de presentarse en todas las circunscripciones electorales, puesto que todos los votos les pueden ser útiles en el reparto a segundo nivel. Por ello, la barrera continua no debiera bajar del $2 \%$ si se desea "no dificultar la gobernabilidad».

\section{MODIFICACIÓN DEL ARTÍCULO 68 DE LA CONSTITUCIÓN}

Es posible establecer una modificación que:

i. No implique una reforma inmediata de la Ley Electoral de 1985.

ii. Mantenga el espíritu de la Constitución en cuanto a: proporcionalidad, circunscripciones, tamaño del Congreso, etc.

iii. Abra posibilidades de reforma de la Ley Electoral que permitan:

a. Obtener un valor muy similar del voto de cualquier ciudadano, sea cual sea su circunscripción, sea cual sea el partido al que vote (siempre que dicho partido no sea muy pequeño).

b. Evite las contradicciones entre votos totales y escaños totales de los partidos. Por supuesto, evite contradicciones entre los dos primeros partidos (como ocurrió en dos elecciones autonómicas catalanas).

c. Aumente la proporcionalidad del sistema electoral.

d. Aumente la participación en las elecciones.

e. Aumente el número de votos útiles en las elecciones generales.

f. Posibilite la aparición de nuevos partidos.

g. No dificulte la gobernabilidad, etc.

Una posible modificación del artículo 68 de la Constitución que permite lograr los objetivos citados anteriormente es la siguiente: 


\section{Artículo 68 modificado:}

Los apartados 2. y 3. podrían substituirse por los siguientes:

2. Para al menos 300 Diputados, de los que constituyan el Congreso, las circunscripciones electorales serán las provincias y cada una de las poblaciones de Ceuta y Melilla. Uno de esos Diputados representará a Ceuta, otro a Melilla y la distribución de los restantes escaños entre las provincias se hará asignado un número inicial fijo a cada provincia y los restantes en proporción a sus poblaciones.

3. Los escaños de las circunscripciones anteriores se asignarán a los partidos que concurran en cada una de ellas atendiendo a criterios de representación proporcional.

Por supuesto que el valor 300 también puede ser otro, por ejemplo 350 como en la actualidad. En cualquier caso, una modificación como la anterior, u otra similar, no obliga a cambiar la Ley Electoral, pero caso de cambiarla se tiene la posibilidad de disponer de un número de escaños libres que permiten realizar un reparto biproporcional o un reparto a dos niveles, o bien otras muchas posibilidades.

$\mathrm{Si}$, por el contrario, se tiene intención de cambiar la Ley Electoral entonces la redacción anterior para modificar el artículo 68 no está obligada a ser tan forzada.

\section{Agradecimientos}

El autor desea agradecer a la Junta de Andalucía por su apoyo a través del grupo FOM-191, que permite sufragar parte de los gastos de la investigación en sistemas electorales. También al Departamento de Matemática Aplicada de la Universidad de Granada por sus ayudas para la investigación. Y a los profesores/as A. Garralda, A. Márquez y a M. Pasadas por sus sugerencias, que han permitido mejorar este trabajo.

\section{REFERENCIAS}

1. BaLINSKI, M. L., Young H. P., Fair Representation: Meeting the Ideal of One Man One Vote. New Haven, CT, 1982.

2. BALINSKI, M. L., Demange., Biproportional apportionment . 
3. MÁrouez, M. L. Representación proporcional. Representación Parlamentaria, Tesis doctoral. Univeridad de Granada, 1997.

4. MÁrouez, M. L., Ramírez, V., The Spanish electoral system: Proportionality and governability, Annals of Operation Research, pp. 45-59, 1998.

5. Ramírez, V., Palomares, A., El tamaño de las circunscripciones para el Congreso, Revista de derecho Político, UNED, n. ${ }^{\circ} 63$, pp. 41-54, Madrid-2005.

6. Pukelsheim, F., Das Kohärenzprinzip, angewandt auf den Deuschen Bundestag, Spektrum, pp. 96-97, Mars, 2004

7. VIDAL C, El sistema electoral español. Una propuesta de reforma. Tesis doctoral. Universidad de Navarra, 1993

8. VIDAL C., El sistema electoral español: una propuesta de reforma. Método Ediciones, Granada, 1995.

9. http://www.elecciones.mir.es/MIR/jsp/resultados/index.htm 


\section{ANEXO}

Resultados globales en las nueve elecciones al Congreso de los Diputados celebradas entre 1977 y 2004.

\section{Elección de 1977}

\begin{tabular}{|l|c|c|c|c|c|}
\hline Partido & Votos totales & \% Votos & Cuota & Escaños & \% Escaños \\
\hline UCD & $6,337,288$ & 37.99 & 132.96 & 166 & 47.43 \\
\hline PSOE & $5,358,781$ & 32.13 & 112.43 & 118 & 33.71 \\
\hline PCE & $1,718,026$ & 10.30 & 36.04 & 21 & 6.00 \\
\hline AP & $1,525,028$ & 9.14 & 31.99 & 18 & 5.14 \\
\hline PSP-US & 816,510 & 4.89 & 17.33 & 6 & 1.71 \\
\hline PDC & 514,647 & 3.09 & 10.80 & 11 & 3.14 \\
\hline PNV & 314,409 & 1.88 & 6.60 & 8 & 2.29 \\
\hline EE & 60,312 & 0.36 & 1.27 & 1 & 0.29 \\
\hline AIC & 37,183 & 0.22 & 0.78 & 1 & 0.29 \\
\hline Totales & $16,682,184$ & 100 & 350 & 350 & 100 \\
\hline
\end{tabular}

\section{Elección de 1979}

\begin{tabular}{|l|c|c|r|r|c|}
\hline Partido & Votos totales & \% Votos & Cuota & Escaños & \% Escaños \\
\hline UCD & $6,262,593$ & 37.05 & 129.68 & 167 & 47.71 \\
\hline PSOE & $5,496,813$ & 32.36 & 113.27 & 123 & 35.14 \\
\hline PCE & $1,938,487$ & 11.47 & 40.14 & 25 & 7.14 \\
\hline CD & $1,064,333$ & 6.30 & 22.04 & 9 & 2.57 \\
\hline CiU & 483,254 & 2.86 & 10.01 & 9 & 2.57 \\
\hline UN & 378,955 & 2.24 & 7.85 & 1 & 0.29 \\
\hline PSA & 325,762 & 1.93 & 6.75 & 5 & 1.43 \\
\hline PNV & 275,292 & 1.63 & 5.70 & 7 & 2.00 \\
\hline PTE & 192,789 & 1.14 & 3.99 & 0 & 0.00 \\
\hline HB & 172,110 & 1.02 & 3.56 & 2 & 0.57 \\
\hline ORT & 127,417 & 0.75 & 2.64 & 0 & 0.00 \\
\hline ERC & 125,690 & 0.74 & 2.60 & 1 & 0.29 \\
\hline EE & 85,677 & 0.51 & 1.77 & 1 & 0.29 \\
\hline Totales & $16,902,181$ & 100 & 350 & 350 & 350 \\
\hline
\end{tabular}




\section{Elección de 1982}

\begin{tabular}{|l|c|r|r|r|c|}
\hline Partido & Votos totales & $\%$ Votos & Cuota & Escaños & \% Escaños \\
\hline PSOE & $10,127,392$ & 50.41 & 176.44 & 202 & 57.71 \\
\hline AP & $5,543,107$ & 27.59 & 96.58 & 107 & 30.57 \\
\hline UCD & $1,354,858$ & 6.74 & 23.61 & 11 & 3.14 \\
\hline PCE & 844,976 & 4.21 & 14.72 & 4 & 1.14 \\
\hline CIU & 772,726 & 3.85 & 13.46 & 12 & 3.43 \\
\hline CDS & 600,842 & 2.99 & 10.47 & 2 & 0.57 \\
\hline PNV & 395,629 & 1.97 & 6.89 & 8 & 2.29 \\
\hline HB & 210,601 & 1.05 & 3.67 & 2 & 0.57 \\
\hline ERC & 138,116 & 0.69 & 2.41 & 1 & 0.29 \\
\hline EE & 100,326 & 0.50 & 1.75 & 1 & 0.29 \\
\hline Totales & $20,088,573$ & 100 & 350 & 350 & 100 \\
\hline
\end{tabular}

\section{Elección de 1986}

\begin{tabular}{|l|c|c|r|c|c|}
\hline Partido & Votos totales & \% Votos & Cuota & Escaños & \% Escaños \\
\hline PSOE & $8,901,718$ & 47.33 & 165.66 & 184 & 52.56 \\
\hline CP & $5,247,677$ & 27.90 & 97.66 & 105 & 30.00 \\
\hline CDS & $1,838,799$ & 9.78 & 34.22 & 19 & 5.43 \\
\hline CIU & $1,014,258$ & 5.39 & 18.87 & 18 & 5.14 \\
\hline IU & 768,158 & 4.08 & 14.29 & 6 & 1.71 \\
\hline PNV & 309,610 & 1.65 & 5.76 & 6 & 1.71 \\
\hline HB & 215,282 & 1.14 & 4.01 & 5 & 1.43 \\
\hline UER & 123,912 & 0.66 & 2.31 & 1 & 0.29 \\
\hline EE & 107,053 & 0.57 & 1.99 & 2 & 0.57 \\
\hline CG & 79,972 & 0.43 & 1.49 & 1 & 0.29 \\
\hline PAR & 71,628 & 0.38 & 1.33 & 1 & 0.29 \\
\hline AIC & 64,989 & 0.35 & 1.21 & 1 & 0.29 \\
\hline UV & 64,403 & 0.34 & 1.20 & 1 & 0.29 \\
\hline Totales & $18,807,459$ & 100 & 350 & 350 & 100 \\
\hline
\end{tabular}




\section{Elección de 1989}

\begin{tabular}{|l|c|c|r|c|c|}
\hline Partido & Votos totales & \% Votos & Cuota & Escaños & \% Escaños \\
\hline PSOE & $8,088,072$ & 42.39 & 148.40 & 175 & 50.00 \\
\hline PP & $5,282,877$ & 27.69 & 96.93 & 107 & 30.57 \\
\hline IU & $1,851,080$ & 9.70 & 33.96 & 17 & 4.86 \\
\hline CDS & $1,617,104$ & 8.48 & 29.67 & 14 & 4.00 \\
\hline CIU & $1,030,476$ & 5.40 & 18.91 & 18 & 5.14 \\
\hline PNV & 253,769 & 1.33 & 4.66 & 5 & 1.43 \\
\hline HB & 216,822 & 1.14 & 3.98 & 4 & 1.14 \\
\hline PA & 212,807 & 1.12 & 3.90 & 2 & 0.57 \\
\hline UV & 144,655 & 0.76 & 2.65 & 2 & 0.57 \\
\hline EA & 136,595 & 0.72 & 2.51 & 2 & 0.57 \\
\hline EE & 105,217 & 0.55 & 1.93 & 2 & 0.57 \\
\hline PAR & 71,628 & 0.38 & 1.31 & 1 & 0.29 \\
\hline AIC & 64,989 & 0.34 & 1.19 & 1 & 0.29 \\
\hline Totales & $19,076,091$ & 100 & 350 & 350 & 100 \\
\hline
\end{tabular}

\section{Elección de 1993}

\begin{tabular}{|l|c|c|c|c|c|}
\hline Partido & Votos totales & \% Votos & Cuota & Escaños & \% Escaños \\
\hline PSOE & $9,071,760$ & 40.52 & 141.81 & 159 & 45.42 \\
\hline PP & $8,170,201$ & 36.49 & 127.71 & 141 & 40.28 \\
\hline IU & $2,245,444$ & 10.03 & 35.10 & 18 & 5.14 \\
\hline CIU & $1,162,534$ & 5.19 & 18.17 & 17 & 4.86 \\
\hline CDS & 414,000 & 1.85 & 6.47 & 0 & 0.00 \\
\hline PNV & 300,308 & 1.34 & 4.69 & 5 & 1.43 \\
\hline CC & 206,953 & 0.92 & 3.24 & 4 & 1.14 \\
\hline HB & 206,296 & 0.92 & 3.22 & 2 & 0.57 \\
\hline PAR & 144,241 & 0.64 & 2.25 & 1 & 0.29 \\
\hline BNG & 126,965 & 0.57 & 1.98 & 0 & 0.00 \\
\hline ERC & 121,972 & 0.54 & 1.91 & 1 & 0.29 \\
\hline EA & 117,859 & 0.53 & 1.84 & 1 & 0.29 \\
\hline UV & 102,714 & 0.46 & 1.61 & 1 & 0.29 \\
\hline Totales & $22,391,247$ & 100 & 350 & 350 & 100 \\
\hline
\end{tabular}




\section{Elección de 1996}

\begin{tabular}{|l|c|c|r|c|c|}
\hline Partido & Votos totales & \% Votos & Cuota & Escaños & \% Escaños \\
\hline PP & $9,716,006$ & 40.09 & 140.33 & 156 & 44.57 \\
\hline PSOE & $9,425,678$ & 38.89 & 136.13 & 141 & 40.28 \\
\hline IU & $2,639,774$ & 10.89 & 38.13 & 21 & 6.00 \\
\hline CIU & $1,151,633$ & 4.75 & 16.63 & 16 & 4.57 \\
\hline PNV & 318,951 & 1.32 & 4.61 & 5 & 1.43 \\
\hline CC & 220,418 & 0.91 & 3.18 & 4 & 1.14 \\
\hline BNG & 220,147 & 0.91 & 3.18 & 2 & 0.57 \\
\hline HB & 181,304 & 0.75 & 2.62 & 2 & 0.57 \\
\hline ER & 162,545 & 0.67 & 2.35 & 1 & 0.29 \\
\hline EA & 115,861 & 0.48 & 1.67 & 1 & 0.29 \\
\hline UV & 81,350 & 0.34 & 1.17 & 1 & 0.29 \\
\hline Totales & $24,233,667$ & 100 & 350 & 350 & 100 \\
\hline
\end{tabular}

\section{Elección de 2000}

\begin{tabular}{|l|c|r|r|r|c|}
\hline Partido & Votos totales & \% Votos & Cuota & Escaños & \% Escaños \\
\hline PP & $10,321,178$ & 46.75 & 163.63 & 183 & 52.28 \\
\hline PSOE & $7,918,752$ & 35.87 & 125.54 & 125 & 35.71 \\
\hline IU & $1,263,043$ & 5.72 & 20.02 & 8 & 2.28 \\
\hline CIU & 970,421 & 4.40 & 15.38 & 15 & 4.28 \\
\hline PNV & 353,953 & 1.60 & 5.61 & 7 & 2.00 \\
\hline BNG & 306,268 & 1.39 & 4.85 & 3 & 0.86 \\
\hline CC & 248,261 & 1.12 & 3.94 & 4 & 1.14 \\
\hline PA & 206,255 & 0.93 & 3.27 & 1 & 0.29 \\
\hline ERC & 194,715 & 0.88 & 3.09 & 1 & 0.29 \\
\hline IC-V & 119,290 & 0.54 & 1.89 & 1 & 0.29 \\
\hline EA & 100,742 & 0.46 & 1.54 & 1 & 0.29 \\
\hline CHA & 75,356 & 0.34 & 1.19 & 1 & 0.29 \\
\hline Totales & $22,078,234$ & 100 & 350 & 350 & 100 \\
\hline
\end{tabular}




\section{Elección de 2004}

\begin{tabular}{|l|c|c|r|r|c|}
\hline Partido & Votos totales & \% Votos & Cuota & Escaños & \% Escaños \\
\hline PSOE & $11,026,163$ & 44.38 & 155.34 & 164 & 46.85 \\
\hline PP & $9,763,144$ & 39.30 & 137.54 & 148 & 42.28 \\
\hline IU & $1,284,081$ & 5.17 & 18.09 & 5 & 1.43 \\
\hline CIU & 835,471 & 3.36 & 11.77 & 10 & 2.86 \\
\hline ERC & 652,196 & 2.63 & 9.19 & 8 & 2.28 \\
\hline PNV & 420,980 & 1.69 & 5.93 & 7 & 2.00 \\
\hline CC & 235,221 & 0.95 & 3.31 & 3 & 0.86 \\
\hline BNG & 208,688 & 0.84 & 2.94 & 2 & 0.51 \\
\hline PA & 181,868 & 0.73 & 2.56 & 0 & 0.00 \\
\hline CHA & 94,252 & 0.38 & 1.33 & 1 & 0.29 \\
\hline EA & 80,905 & 0.33 & 1.14 & 1 & 0.29 \\
\hline NA-BAI & 61,045 & 0.24 & 0.86 & 1 & 0.29 \\
\hline Totales & $24,5844,014$ & 100 & 350 & 350 & 100 \\
\hline
\end{tabular}

Title:

"Constitutional limitations concerning the election of the congress of deputies. Possible modification of article 68 of the Spanish Constitution and allotment at two levels".

\section{Summary}

0. INTRODUCTION. 1. CURRENT CONSTITUTIONAL LIMITATIONS. 2. ELECTORAL LAW AND PRINCIPAL CONTRADICTIONS IN THE GLOBAL RESULTS IN ELECTIONS OF THE CONGRESS OF DEPUTIES. 3. VOTES THAT HAVE NOT GIVEN RISE TO REPRESENTATION IN THE ELECTION OF 2004. 4. POSSIBILITIES FOR REFORM OF THE ELECTORAL LAW WITHIN THE CURRENT CONSTITUTIONAL FRAMEWORK. 5. ALLOTMENT AT TWO LEVELS. THE BUNDESTAG ELECTION IN GERMANY. 6. WHAT RESULTS MIGHT HAVE BEEN OBTAINED IN THE ELECTION OF THE CONGRESS IN 2004 USING AN ALLOTMENT AT THE SECOND LEVEL? 7. MODIFICATION OF ARTICLE 68 OF THE CONSTITUTION. REFERENCES.

\section{Resumen}

La Constitución de 1978, en los artículos 9 y 14, establece la igualdad de los individuos y de los grupos en que se integran, sin que pueda 
prevalecer discriminación alguna por razón de opinión u otra circunstancia personal. Sin embargo, en el artículo 68, establece limitaciones relativas al sistema electoral para la elección del Congreso de los Diputados que conducen a diferencias importantes del valor del voto de los ciudadanos dependiendo del partido al que se vote y la circunscripción a la que se está adscrito.

Este trabajo muestra los principales agravios que produce la elección del Congreso cuando se comparan votos totales y escaños totales recibidos por los diferentes partidos políticos. En muchas ocasiones un partido con más votos que otro recibe menos escaños que ese otro. Siempre resultan perjudicados los partidos de ámbito estatal al compararlos con los partidos de ámbito autonómico que han obtenido similar número de votos.

Por otra parte, se recoge el concepto de asignación de escaños a dos niveles (condicionando el reparto a segundo nivel a los resultados obtenidos en el reparto a primer nivel), como se hace para la elección del Bundestag en Alemania, y se resaltan las ventajas de ese sistema. A continuación se muestra un reparto a dos niveles para la elección del Congreso usando los datos electorales de 2004, sin necesidad de diseñar distritos uninominales, como en Alemania, sino usando exclusivamente como circunscripciones las provincias (además de las ciudades de Ceuta y Melilla). Con el reparto a dos niveles los principales agravios, que se han producido en las nueve elecciones generales celebradas entre 1977 y 2004, desaparecen y, al mismo tiempo, aumenta la proporcionalidad del sistema electoral sin dificultar la gobernabilidad.

Finalmente, con objeto de que un reparto a dos niveles pueda recogerse, si se desea, en una modificación de la Ley Electoral de 1985 y esté garantizada la constitucionalidad de dicha reforma, se propone una ligera modificación del artículo 68 de la Constitución. Dicha modificación no obliga a cambiar la Ley Electoral actual, porque simplemente debilita algunas de las limitaciones establecidas en dicho artículo.

\title{
Palabras clave:
}

Sistema Electoral. Proporcionalidad. Repartos a varios niveles.

\begin{abstract}
The Spanish Constitution of 1978, in its articles 9 and 14, establishes the equality of individuals and of the groups that they belong to where
\end{abstract}


no discrimination can prevail because of opinion or personal circumstance. Nevertheless, article 68 establishes limitations relative to the electoral system for the election of the Congress of Deputies that lead to important differences in the value of citizens' votes depending on the party voted for and the circumscription to which the voter is assigned.

This work shows the principal damage that the election of the Congress produces when total votes and total seats obtained are compared for the different political parties. In many occasions a party with more votes than another receives fewer seats. The political parties at the national level always suffer at the expense of the parties of a regional or autonomous nature that have obtained similar numbers of votes.

We also present the concept of assignment of seats at two levels (determining the allotment at the second level in view of the results obtained in the allotment at the first level), as is done at the election of the Bundestag in Germany, highlighting the advantages of this system. A possible allotment at two levels is then shown for the election of the Congress using the electoral information of 2004, with no need to design unipersonal districts, as in Germany, but using exclusively as circumscriptions the Spanish provinces (along with the cities of Ceuta and Melilla). With the allotment at two levels, the principal damage, produced in nine general elections celebrated between 1977 and 2004, would disappear and, at the same time, the proportionality of the electoral system would increase without impeding governability.

Finally, we propose that an allotment at two levels could be established, if desired, through a modification of the Electoral Law of 1985 introducing a slight modification of article 68 of the Constitution in order to guarantee the constitutionality of the proposed reform. The above mentioned modification does not force change in the current Electoral Law, because it simply weakens some of the limitations established in the article 68.

\section{Key words:}

Electoral System. Proportionality. Allotments at several levels. 